\title{
Geliştirilmiş EEMD-EWT Tabanlı Yapay Sinir Ağı Modeli Kullanarak Çok Adımlı Rüzgar Hızı Tahmini
}

\author{
Cem Emeksiz ${ }^{1 *}$, Mustafa Tan ${ }^{2}$

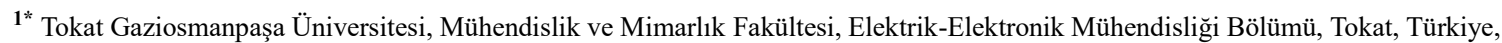 \\ (ORCID: 0000-0002-4817-9607), cem.emeksiz@gop.edu.tr \\ 2 Tokat Gaziosmanpaşa Üniversitesi, Mühendislik ve Mimarlık Fakültesi, Elektrik-Elektronik Mühendisliği Bölümü, Tokat, Türkiye, \\ (ORCID: 0000-0002-5820-6613), mustan79@gmail.com
}

(3rd International Congress on Human-Computer Interaction, Optimization and Robotic Applications June 11-13, 2021)

(DOI: 10.31590/ejosat.948661)

ATIF/REFERENCE: Emeksiz, C. \& Tan, M., (2021). Geliştirilmiş EEMD-EWT Tabanlı Yapay Sinir Ağı Modeli Kullanarak Çok Adımlı Rüzgar Hızı Tahmini. Avrupa Bilim ve Teknoloji Dergisi, (26), 165-173.

\section{$\ddot{O} \mathbf{z}$}

Rüzgar hızı tahminlemesi rüzgar güç dönüşüm sistemleri için oldukça önemlidir. Bu çalışmada kısa vadeli rüzgar hızı tahminlemesi için hibrit bir ayrıklaştırma yöntemi önerilmiştir. Önerilen yöntemde Toplu ampirik mod ayrıştırma (Ensemble Empirical Mode Decomposition, EEMD) ve Ampirik dalgacık dönüşümü (Emprical wavelet transform, EWT) birlikte kullanılmıştır. İlk defa kullanılan bu kombinasyon sonucunda elde edilen ayrıklaştırılmış rüzgar hızı sinyalleri kısmi otokorelasyon fonksiyonu (Partial autocorrelation function ${ }_{2}$ PACF) ile öznitelik çıkarma işlemine tabi tutulmuştur. Elde edilen öznitelikler, geri beslemeli sinir ağına (Back propagation neural networks, BPNN) uygulanmak suretiyle çok adımlı rüzgar hız tahminleme işlemi gerçekleştirilmiştir. Önerilen modelin birbirinden bağımsız teknikler kullanılarak yapılan tekil ayrıklaștırmaya göre çok daha doğru ve güvenilir sonuçlar verdiği tespit edilmiştir. Çalışmada kullanılan veriler Tokat Gaziosmanpaşa Üniversitesi Taşlıçiftlik Kampüsü içerisinde kurulan ölçüm istasyonundan toplanmıștır. Önerilen hibrit model, yüksek hassasiyetli rüzgar hızı tahminleri için güvenilir, güçlü ve etkili olduğu kadar veri madenciliği uygulamalarında da kolaylıkla kullanılabilir. Tahmin performansının genel tahmin doğruluğu yaygın olarak kullanılan üç genel hata değerlendirme endeksi olan determinasyon katsayısı (determination coefficient $\left(\mathrm{R}^{2}\right)$, ortalama mutlak yüzde hata (mean absolute percent error (MAPE) ve ortalama karekök hata (root mean square error (RMSE)) ile gerçekleştirildi.

Anahtar Kelimeler: Rüzgar hızı tahmini, Hibrit model, Topluluk ampirik mod ayrıştırma, Ampirik dalgacık dönüşümü, Geri beslemeli sinir ăg.

\section{Multi-Step Wind Speed Estimation Using Improved EEMD-EWT Based Artificial Neural Network Model}

\begin{abstract}
Wind speed estimation is very important for wind power conversion systems. In this study, a hybrid discretization method is proposed for short-term wind speed estimation. In the proposed method, Ensemble Empirical Mode Decomposition (EEMD) and Empirical wavelet transform (EWT) are used together. Discretized wind speed signals obtained as a result of this combination used for the first time were subjected to feature extraction process with partial autocorrelation function (PACF). The multi-step wind speed estimation process has been carried out by applying the obtained features to the feedback neural network (Back propagation neural networks, BPNN). It has been determined that the proposed model gives much more accurate and reliable results than the singular discretization using independent techniques. The data used in the study were collected from the measurement station established in Tokat Gaziosmanpaşa University Taşliçiftlik Campus. The proposed hybrid model is reliable, powerful and effective for high precision wind speed predictions, as well as easily used in data mining applications. The overall prediction accuracy of the prediction performance was achieved with the three commonly used general error rating indices: determination coefficient ( $\left.\mathrm{R}^{2}\right)$, mean absolute percent error (MAPE) and root mean square error (RMSE).
\end{abstract}

Keywords: Wind speed prediction, Hybrid model, Ensemble empirical mode decomposition, Empirical wavelet transform, Feedback neural network

\footnotetext{
*Sorumlu Yazar: cem.emeksiz@gop.edu.tr
} 


\section{Giriş}

Rüzgar enerjisinden elektrik enerjisi üretimi ve sürdürülebilirliği son yıllarda aktif çalışma konusu olarak önem kazanmaktadır. Rüzgar enerjisi meteorolojisi, temel olarak bölgesel rüzgar potansiyeli analizi, rüzgar türbinlerinin yerleşimi ve kısa süreli rüzgar enerjisi tahminleri ile ilgilenmektedir (Tan, 2020). Rüzgar enerjisi tahmininde en önemli girdi rüzgar hızı olup, rüzgar hızı tahmini rüzgar enerjisi tahmini içinde önemli bir etkiye sahiptir (Guo ve ark., 2012). Elektrik şebeke sistemindeki giriş ve çıkış gücünün dengelenmesinde rüzgar hızının kaotik davranışından dolayı zorlanılmaktadır. Ayrıca, rüzgar türbinlerinin değişken rüzgar hızı nedeniyle zarar görebilme ihtimalleri de vardır. Dolayısıyla, rüzgar enerjisi üretim sistemleri için güvenilir ve kesin rüzgar hızı tahmini hayati önem taşımaktadır. Yapılan bu tahminler, enerji şebekesi dengeleme kaynaklarının planlanmasında uygulanmaktadır. Çalışmalarda, rüzgar enerjisi tahminlerinin geliştirilmesi ile önemli ekonomik ve teknik avantajlar elde edileceği de bildirilmektedir (Qian ve ark., 2019; Zhang ve ark., 2017; Liu ve ark., 2018; Şenkal, 2014).

Genel olarak rüzgar hızı ve rüzgar gücü zaman serilerinin durağan olmadıkları ve güçlü dalgalanmalar içerdikleri bilinmektedir (Aghajani ve ark., 2016). Rüzgar hızı tahmin modellerinin başarısını artırmak amacıyla, son yıllarda literatürde, çok kısa, kısa ve uzun dönem rüzgar hızı veya rüzgar gücü tahminleri sağlamaya yönelik birçok yaklaşım geliştirilmiş ve bildirilmiştir (Zhang ve ark., 2017). Rüzgar hızı tahmini için kullanılan teknikler fiziksel yaklaşım, istatistiksel yaklaşım ve hibrit yaklaşım olarak üç ana gruba ayrılmaktadır. Fiziksel yaklaşım, sayısal hava tahminleri (SHT) gibi hava istasyonlarından elde edilen geçmiş verileri kullanır. Bunların modellenmesi karmaşık olduğundan uzun vadeli tahminler için uygundur. İstatistiksel yaklaşımlar ise Otoregresif Hareketli Ortalama (Autoregressive Moving Average, ARMA) modeli, ARMA türevleri ve Yapay Sinir Ağ1 (YSA) modelleri gibi gelecekteki değerleri modellemek ve tahmin etmek için geçmiş zaman serisi verilerini kullanır. Bu yaklaşımlar, kısa vadeli tahminler içinde kullanılır. Hibrit yaklaşımlar, iki veya daha fazla tahmin yönteminin bir arada kullanılmasıyla elde edilmektedir (Qian ve ark., 2019; Santhosh ve ark., 2018; He ve ark., 2018). Hibrit modeller farklı metodolojilerden yararlanıp ve daha hassas tahmin performansı gösterebildiklerinden, hibrit modellere olan ilgi son zamanda giderek artmıştır (Liu ve ark., 2018). Aynı zamanda yapay zeka tekniklerindeki gelişmeler YSA modellerinin hibrit modellerle kullanılmasını teşvik etmektedir. Bu YSA modelleri, ağırlıklı olarak Geri Yayılım Sinir Ağını (BPNN) içeren, doğrusal olmayan verileri başarıyla işleme yetenekleri nedeniyle rüzgar hızı tahmininde yaygın olarak kullanılmaktadır (Santhosh ve ark., 2018).

Hibrit modellerin oluşturulmasında iki temel yaklaşım kullanılmaktadır. $\mathrm{Bu}$ yaklaşımlardan ilki Parçacık Sürü Optimizasyonu (PSO) (Jiang ve Li, 2018; Ren ve ark., 2014), Genetik Algoritma (GA) (Wang ve ark., 2016) vb. gibi sezgisel optimizasyon algoritmalarının kullanıldığı algoritma temelli yaklaşımdır. İkinci yaklaşım ise orijinal zaman serisine çeşitli ayrıştırma teknikleri uygulamasıdır. Ayrıca hem ayrıştırma tekniği hem de farklı algoritma kombinasyonlarının bir arada kullanıldığ yapılara da rastlamak mümkündür. Rüzgar hızı tahminlemesinde Varyasyon Modu Ayrıştırma (VMD), Toplu Ampirik Mod Ayrıştırma (EEMD), Ampirik Dalgacık Dönüşümü ve Dalgacık Dönüşümü (WT) gibi birçok ayrıştırma tekniği kullanılmaktadır.
Ayrıştırma yöntemlerine dayanan teknikte, orijinal zaman serileri farklı alt dizilere ayrıştırılabilir ve orijinal zaman serilerinden daha etkili bir şekilde tahminleme yapan bir model oluşturulur. (Tascikaraoglu ve Uzunoglu, 2014). Popüler olarak, sıklıkla kullanılan tek ayrıştırma yöntemlerine ek olarak, yakın dönemde ikincil ayrıştırma yöntemleri de araştırılmış ve genellikle tek ayrıştırma yöntemlerinden daha iyi performans sergilediği belirlenmiştir (Peng ve ark., 2017). Tekli zaman serisi ayrıştırma tekniklerinin çoğunda tahminleme yapılmadan önce eğitim veri seti ve test veri seti dahil olmak üzere tüm ham seri, sadece bir kez ayrıştırılmıştır. Ayrıştırma neticesinde elde edilen birinci içsel mod fonksiyou (The intrinsic mode function, IMF), anti-kalıc1 davranış özelliği ile ana veriye olan benzerliğinden dolayı çalışmalarda ikincil ayrıştırmayı birinci IMF üzerinden yapılması yönünde etkilemektedir. Birinci ve ikinci ayrıştırma sonucu elde edilen alt fonksiyonlar, ana verinin özelliklerini yansıtmakla beraber daha durağan bir fonksiyon olarak tahminleme performansı üzerinde etkilidirler (Xiao ve ark., 2015).

Santhosh ve ark. (2018), EEMD tekniğini ve Geliştirilmiş dalgacık sinir ağı (Advanced Wavelet Neural Networks, AWNN) modelini birleştiren hibrit EEMD-AWNN yaklaşımı geliştirmişlerdir. Hibrit EEMD-AWNN modeli rüzgar hızının gelecekteki değerlerini tahmin etmek için kullanarak, önerilen EEMD-AWNN modelinin gelişmiş tahmin doğruluğu ile istenen sonucu elde edebileceğini kanıtlamışlardır (Santhosh ve ark., 2018). Li ve ark. (2018), Ampirik Mod Ayrıştırma (EMD)'nın kullanıldığı BPNN, Elman Sinir Ağı (ENN) ve Otomatik Regresif Entegre Hareketli Ortalama (Auto-Regressive Integrated Moving Average, ARIMA)'ya dayalı bir hibrit model oluşturmuşlardır (Liu ve ark., 2018). Zhang ve ark. (2017), Komple Uyarlamalı Gürültülü Toplu Ampirik Mod Ayrıştırma (EEMD), Kaotik Yerel Arama ile Çiçek Tozlaşma Algoritması (Chaotic Local Search Flower Pollination Algorithm, CLSFPA), beş sinir ağı ve Negatif Kisitlama Yok Teorisi (No Negative Constraint Theory, NNCT)'ne dayalı bir rüzgar hızı tahmin yöntemi sunmuşlardır. Sonuçta, önerilen birleşik modelin tek sinir ağı modelleri ve ARIMA modeli ile karşılaştırılarak en iyi performansa sahip olduğunu göstermişlerdir (Zhang ve ark., 2017). Emeksiz ve Demir (2018) rüzgar enerjisinin en önemli girdileri olarak kabul edilen rüzgar hızlarını makine öğrenme algoritmaları kullanılarak tahmin etmişlerdir. Tahminlemede altı farklı algoritma kullanmış ve bu algoritmalar arasında en düşük hata ve en yüksek korelasyon katsayısı (CC) ile en iyi tahmini Bagging algoritmasında gerçekleştirmişlerdir. Çalışmada rüzgar hızı tahminini etkileyen meteorolojik parametreleri de incelemişler ve sıcaklık-nem-basınç kombinasyonunun daha düşük hata oranlı bir tahmin gerçekleştirdiğini göstermişlerdir (Emeksiz ve Demir, 2018). He ve ark. (2018) çalışmalarında güçlü bir hibrit sistem geliştirmişlerdir. Veri ön işleme modülünde EEMD tekniği ve veri kümeleme modülü olarak Çekirdek Tabanlı Bulanık C-Ortalamalı Kümeleme (Kernel-Based Fuzzy C-Mean Clustering, KFCM) algoritması ve tahminleri yürütmek için Dalgacık Sinir Ağı (WNN) modeli oluşturmuşlardır (He ve ark., 2018). Wang ve ark. (2016), rüzgar hızı tahmininde hibrit yöntem EEMD-GA-BP'nin geleneksel GA-BP modelinden daha doğru olduğunu göstermişlerdir (Wang ve ark., 2016). Hu ve ark. (2015), orijinal veri serilerinin farklı frekanslara sahip belirli sinyallere ayrıştırıldığ1 ve ardından tahmin için Gri Destek Vektör makinesinin (GSVM) kullanıldığı EEMD'ye dayalı bir hibrit tahmin modeli önermişlerdir (Hu et al., 2015). He ve ark., (2018), orijinal rüzgar hızı veri setlerini bir dizi bağımsız İçsel Mod Fonksiyonuna (IMF) ayırmak ve farklı frekanslardaki IMF'lerin değerlerini tahmin etmek için SVM'yi kullanmak için EEMD'ye 
dayalı bir hibrit yöntem önermişlerdir (He ve ark., 2018). Torres ve ark. (2011), EEMD'ye dayalı yeni bir modifikasyon yöntemi olan uyarlanabilir gürültülü tam toplu ampirik mod ayrışımını (EEMD) önermişlerdir (Torres ve ark., 2011). Qian ve ark. (2019), ayrışma tabanlı hibrit yöntemler açısından rüzgar enerjisi tahmin literatürünü gözden geçirmişlerdir. Daha spesifik olarak, ayrışmaya dayalı modelleri özetleyerek, yapılarını ve ilişkili algoritmalarını tartışmışlardır. Ayrıştırma tabanlı hibrit modellerin tahmin doğruluğunu nasıl iyileştirdiğini incelemişler ve performansını artırmak için son yıllarda benimsenen mevcut teknikler de özetlemişlerdir (Qian ve ark., 2019). Gou ve ark. (2012) çalışmalarında EMD tabanlı yapay sinir ağını modifiye edilerek bir model geliştirmişlerdir. Çalışmalarının çekirdeği olan Değiştirilmiş EMD tabanlı FNN modeli gösterilmektedir. Ayrıca, IMF1'den kurtulmadan önerdikleri temel iki modelin, tahmin doğruluğunu için çok adımlı rüzgar hızı tahmini yapmışlar ve modelin verimliliğini göstermişlerdir (Guo ve ark., 2012). Liu ve ark. (2015), tekli ve ikincil ayrıştırma için birçok karşılaştırmalı vaka çalışması yapmışlardır. Vaka sonuçlarında ikincil ayrıştırmanın, çok aşamalı rüzgar hızı tahmininde tekli modelden daha iyi performans gösterdiğini göstermişlerdir. Bununla birlikte, ikincil ayrıştırma prosedürü, tekli ayrıştırmadan çok daha fazla hesaplama kaynağı gerektirdiğini de vurgulamışlardır (Liu ve ark., 2015).

$\mathrm{Bu}$ çalışmanın bilimsel katkıları ve yenilikçi yönleri ise şu şekilde sıralanmaktadır: (1) Rüzgar hızının yüksek doğruluk ve güvenilirlikte tahminlenmesi için EEMD - EWT - PACF - BPNN adlı yeni bir adaptif dinamik hibrit model önerildi. (2) EEMD sonucu oluşan IMF'ler arasında IMF1 en düzensiz ve doğrusal olmayan parça olduğundan ikincil ayrıştırmada tahmin doğruluğunu artırmak için yüksek frekanslı IMF1 üzerinde EWT kullanılmıştır. (3) Önerilen modelle BPNN'ün tahmin doğruluğu ve kararlılığı da artırıldı. (4) Çalışmanın diğer yenilikçi ve önemli noktası ise BPNN girdi seçiminde kullanılmak üzere elde edilen tüm alt fonksiyonlar PACF kullanılarak öznitelikleri çıkarılmıştır. Böylece tek ölçekte yapılan araştırmalara göre daha doğru tahminleme yapıldığı gibi rüzgarın rastgelelik davranış özelliği de ortaya konmuştur.

Sunulan çalışmanın geri kalan bölümleri şu şekilde düzenlenmiştir. Bölüm 2 önerilen hibrit tahminleme modelinde kullanılan EEMD, EWT ve PACF yöntemleri ayrıntılı olarak açıklamaktadır. Bölüm 3'te çalışmada kullanılan veriler ile ilgili genel bilgiler verilmiştir. Bölüm 4'te rüzgar hızı tahminlemesi önerilen hibrit model ile gerçekleştirilip tahmin sonuçları ve model performansı sunulmuştur. Son bölümde ise yapılan çalışmanın sonuçları özetlenmektedir.

\section{Materyal ve Metot}

Çalışmada kullanılan EEMD, EWT ve PACF yöntemleri ve bunların birleştirilmesi neticesinde elde edilen geliştirilmiş EEMD-EWT tabanlı yapay sinir ağı modeli detaylı bir şekilde aşağıdaki bölümlerde açıklanmaktadır.

\subsection{EEMD (Toplu ampirik mod ayrıştırma)}

Zaman serisi EMD tekniğini kullanarak bir dizi IMF'ye ayrıştırılabilir. Her IMF'de sadece bir salınım modu vardır ve şu iki temel prensibi karşılar (Gilles, 2013) (Huang ve ark. 1998). İlk prensip toplam veri setinde, sıfır geçiş sayısının ve uç nokta sayısının eşit ya da farklı olması gerektiğini belirtir. İkincisi ise, IMF'nin herhangi bir noktasinda, yerel minimum ve yerel maksimum tarafindan tanımlanan zarfların ortalama değeri sıfırdır. EMD’ye kıyasla sahip olduğu avantajlar sayesinde EEMD işaret işleme teknikleri arasında yaygın olarak tercih edilmekte ve kullanılmaktadır. EMD, aynı ve farklı mod da bulunabilen çeşitli salınım türleri gibi karışık mod özelliği sergiler. EEMD, klasik EMD'nin mod karıştırma problemini ortadan kaldırmak için Wu ve Huang tarafından geliştirilmiştir (Wu ve Huang, 2009). EEMD yönteminin özü, EMD yöntemine yardımcı olmak, frekans ölçeklerinin doğal bir şekilde ayrılmasını kolaylaştırmak ve sonuç olarak mod karıştırma oluşumunu azaltmak için tüm zaman-frekans boşluğunda eşit şekilde düşen beyaz gürültünün eklenmesidir. Sağladığı avantajlar göz önünde bulundurulduğunda ilk ayrıştırma ayrıştırma işleminde EEMD tekniği tercih edilmiştir. EEMD yaklaşımının prosedürleri aşağıdaki şekilde sıralanmaktadır (Sun ve ark., 2018):

1.adım: Topluluk sayısı (En) ve beyaz gürültünün genliğini belirlenir.

2.adım: Orijinal zaman serisi $x(n)$ 'ye beyaz gürültü serisi eklenir ve ardından yeni bir zaman serisi elde edilmiş olur. $X_{i}(n)=x(n)+$ noise $_{i}(n)$

3.adım: Yeni zaman serisi $X_{i}(n)$ standart EMD yöntemini kullanarak birkaç iç mod işlevine (IMF) ve bir artık seriye ayrıştırılır:

$$
X_{i}(n)=\sum_{j=1}^{n} I M F_{i j}(n)+R_{i}(n)
$$

Burada $I M F_{i j}(n)$ ve $R_{i}(n)$ sirasiyla i-inci deneme sirasinda jinci IMF ve artık seriyi temsil eder. n, ayrıştırılmış IMF modlarının toplam sayısıdır.

4.adım: 2. ve 3. adım, $E_{n}$ kez tekrarlanır ve her yinelemede farklı gürültü serileri eklenir

5.adım: $E_{n}$ denemede nihai ayrıştırma sonucu olarak her IMF ve kalıntı için denemelerin topluluk ortalamasını hesaplanır;

$$
\overline{I M F_{j}(n)}=\frac{1}{E n} \sum_{i=1}^{E n} I M F_{i j}(n),
$$

$\overline{\mathrm{R}}$ Hata! Yer işareti tanımlanmamış. $=\frac{1}{E n} \sum_{i=1}^{E n} R_{i}(n)$

Ek olarak (He ve ark., 2018)'e göre, topluluk sayısı M = 100 olarak ayarlanırken eklenen gürültü serisinin standart sapması 0.2 'ye eşittir.

\subsection{EWT (Ampirik dalgacık dönüşümü)}

EWT, Jerome Gilles tarafından önerilen yeni bir sinyal işleme tekniğidir (Gilles, 2013). EWT, analiz edilen sinyalin spektrum bilgisine bağlı olan bir grup geçiş filtresi oluşturur. Analiz edilen sinyalin Fourier desteği $[0, \pi] \mathrm{N}$ bitişik segmente ayrılır. Her bir segment $\Lambda_{n}=\left[\omega_{n-1}, \omega_{n}\right]$ olarak ifade edilir. $\omega_{n}$ ayırıcıdır. Ampirik ölçekleme fonksiyonu ve ampirik dalgacıklar sırasıyla Denklem 1 ve 2 kullanılarak hesaplanır.

Bununla birlikte sunulan algoritmanın geçerliliğinin korunması için bazı kısıtlamalar vardır. Örneğin, 3 ve 4 no'lu denklemde yer alan $\gamma$ oranı ampirik ölçekleme fonksiyonunu ve ampirik dalgacıkları $L^{2}(R)$ 'nin dar bir ölçeği olarak $\gamma<\min _{n}\left(\omega_{n+1}-\omega_{n}\right) /\left(\omega_{n+1}+\omega_{n}\right)$ gibi küçük bir değerle sinırlandirılır. $\beta(x)$ genellikle $\beta(x)=x^{4}\left(35-84 x+70 x^{2}-\right.$ $\left.20 x^{3}\right)$ ile tanımlanır. Orijinal sinyal, aşağıdaki gibi tanımlanan çeşitli ampirik modlara ayrıştırılabilir. 


$$
\begin{aligned}
& \widehat{\Phi_{n}}(\omega)=\left\{\begin{array}{c}
1 \\
\operatorname{Cos}\left[\frac{\pi}{2} \beta\left(\frac{1}{2 \gamma \omega_{n}}\left(|\omega|-(1-\gamma) \omega_{n}\right)\right)\right] \\
0
\end{array}\right. \\
& w_{f}^{\varepsilon}(0, t)=\left\langle f, \emptyset_{1}\right\rangle=\int f(\tau) \overline{\emptyset_{1}(\tau-t)} d \tau=\left(\hat{f}(\omega) \overline{\overline{\left.\emptyset_{1}(\omega)\right)^{v}}}\right. \\
& w_{f}^{\varepsilon}(n, t)=\left\langle f, \psi_{n}\right\rangle=\int f(\tau) \overline{\psi_{n}(\tau-t)} d \tau=\left(\hat{f}(\omega) \overline{\left.{\overline{\psi_{n}}}(\omega)\right)^{\mathrm{v}}}\right. \\
& f_{0}(t)=w_{f}^{\varepsilon}(0, t) * \emptyset_{1} \\
& f_{k}(t)=w_{f}^{\varepsilon}(k, t) * \psi_{k}
\end{aligned}
$$

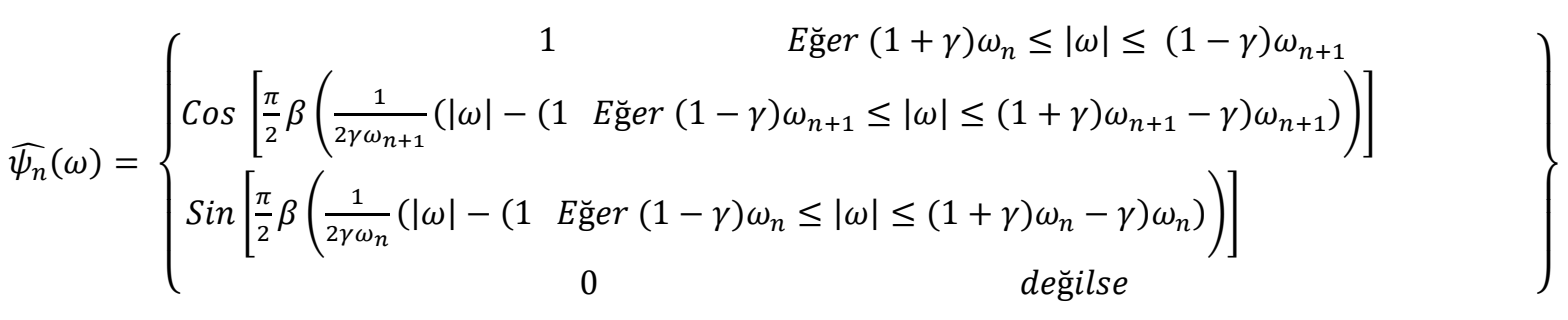$$
\left.\begin{array}{c}
E \breve{\mathrm{g} e r}|\omega| \leq(1-\gamma) \omega_{n} \\
\text { Ĕger }(1-\gamma) \omega_{n} \leq|\omega| \leq(1+\gamma) \omega_{n} \\
\text { değilse }
\end{array}\right\}
$$

\subsection{Geliştirilmiş EEMD-EWT-PACF-BPNN modeli}

Doğrusal ve durağan olmama özelliklerine sahip orijinal rüzgar hızı serisi tahminlemesi hakkında derinlemesine bir kavrayış için, rüzgar hızı serisi önce EEMD tarafından çeşitli frekanslarda alt bölümleri olan sonlu sayıda içsel mod fonksiyonlarına (IMF'ler) ayrıştırılır. Orijinal seriden elde edilen ve orijinal seriye en yakın olan IMF1 üzerinden ikincil ayrıştırma işlemini EWT ile yapılır. Daha sonra alt serileri sırasıyla BPNN ile modellenir ve böylece bu alt serilerin eğilimleri tahmin edilebilir. Son olarak tahmin sonucu üretmek için tüm alt serilerin tahmin sonuçları toplanır. BP algoritması denetimli bir öğrenmedir. Tahminleme ve sinıflandırma uygulamalarında bilinen giriş ve hedef çıktı veri setlerini kullanır. Yapı temelde bir giriş katmanı, bir çıkış katmanı ve bir veya daha fazla gizli katmanlardan oluşur. Giriş katmanından başlamak suretiyle tüm katmanlar sırasıyla gizli katmanlara ve çıkış katmanına bağlanır. Her katman bir veya daha fazla nöron içermektedir. Nöronlar arası bağlantılar ağırlıklar içerir. BP'nin işleyiş amacı öğrenme sürecindeki genel çıktı hatasını kademeli bir şekilde en aza indirgemektir. Doğru çıktıyı tahmin etmek için eğitim verileri tekrarlı bir şekilde girdi katmanına uygulanır. BP temelde iki işlemden oluşur: ileri ve geriye doğru işlem (Ozbay ve Karlik, 2002):

Çalışmamızda, $\operatorname{ARMA}(p, q)$ modelinde $p$ parametresinin tanımlanmasından esinlenerek BP'nin girdi değişkenlerini belirlemek için kısmi otokorelasyon fonksiyonunu (PACF) ve PACF'a ait kısmi otokorelasyon grafiğini kullanıldı (Cadenas ve Rivera, 2009). Somut olarak, $X_{i}^{\prime}$ 'nin çıktı değişkeni olduğunu varsayarsak, $\mathrm{k}$ gecikmesindeki kısmi otokorelasyon yaklaşı olarak $(-1.96 / \sqrt{N} ; 1.96 / \sqrt{N})$ olan $\% 95$ güven aralığının dışındaysa, $X_{i-k}$ girdi değişkenlerinden biridir. Bazen tüm PACF katsayılarının \%95 güven aralığında olduğuna dikkat etmek gerekir. Böyle bir durumda girdi değişkeni olarak önceki bir değeri yani $X_{i-1}$ 'i alıyoruz.

Burada PACF'nin tanımı aşağıdaki gibidir (Wang ve Zhao, 2009). Bir zaman serisi $\left[W_{1}, W_{1}, \ldots, W_{n}\right]$ için $\gamma_{k}$ ile gösterilen $\mathrm{k}$ gecikmesindeki kovaryans ( $\mathrm{k}=0$ ise Varyansdır) denklem. (9)'de gösterilmiştir.

$$
\gamma_{k}=\frac{1}{n} \sum_{i=1}^{n-k}\left(W_{i}-\overline{\mathrm{W}}\right)\left(W_{i+k}-\overline{\mathrm{W}}\right), \mathrm{k}=0,1, \ldots, \mathrm{M}
$$

Burada $\overline{\mathrm{W}}$ serinin ortalamasıdır, $\mathrm{M}=\mathrm{n} / 4$ maksimum gecikmedir. Daha sonra $\rho_{k}$ ile gösterilen $\mathrm{k}$ gecikmesindeki ACF (otokorelasyon fonksiyonu), denklem. (10)'e göre tahmin edilebilir. $\rho_{k}$

$$
\rho_{k}=\frac{\gamma_{k}}{\gamma_{0}}
$$

Kovaryans ve ACF sonucuna dayanarak, $\alpha_{k k}$ ile gösterilen $\mathrm{k}$ gecikmeli PACF hesaplamasını aşağıdaki gibi gösterilir,

$$
\left.\begin{array}{l}
\alpha_{k k}=\rho_{1} \\
\alpha_{k+1, k+1}=\frac{\rho_{k+1}-\sum_{j=1}^{k} \rho_{k+1-j} \alpha_{k j}}{1-\sum_{j=1}^{k} \rho_{j} \alpha_{k j}} \\
\alpha_{k+1, j}=\alpha_{k, j}-\alpha_{k+1, k+1} \alpha_{k, k+1-j}(\mathrm{~J}=1,2, \ldots, \mathrm{k})
\end{array}\right\}
$$

Burada $\mathrm{k}=1,2, \ldots ., \mathrm{M}{ }^{`}$ dir.

\subsection{Performans değerlendirme kriterleri}

Tahmin performansının genel tahmin doğruluğu yaygın (determinasyon katsayısı $\left(\mathrm{R}^{2}\right)$, ortalama mutlak yüzde hata (MAPE) ve kök ortalama kare hatası (RMSE)) ile gerçekleştirildi. $\mathrm{Bu}$ endekslere ait denklemler aşağıdadır (Sun ve ark., 2018):

$$
\begin{aligned}
& \text { RMSE }=\sqrt{\frac{1}{N} \sum_{i=1}^{N}\left(y_{i}-\hat{x}_{i}\right)^{2}} \\
& M A P E=\frac{1}{N} \sum_{i=1}^{N}\left|\frac{\left(y_{i}-\hat{x}_{i}\right)}{y_{i}}\right| \\
& R^{2}=\left(\frac{\sum(x y)-\frac{\left(\sum x\right)\left(\sum y\right)}{n}}{\sqrt{\left[\sum x^{2}-\frac{\left(\sum x^{2}\right)}{n}\right]\left[\sum y^{2}-\frac{\left(\sum y^{2}\right)}{n}\right]}}\right)^{2}
\end{aligned}
$$
olarak kullanılan üç genel hata değerlendirme endeksi 
Denklemlerdeki $y_{i}$ ve $\hat{x}_{i}$ sırasıly i'inci gözlenen ve tahmin edilen rüzgar hızını, $\mathrm{N}$, toplam örnek sayısını temsil etmektedir.

\section{3. Ölçüm İstasyonu ve Veri Toplama}

Çalışmamızda kullanılan rüzgar hızı verileri Tokat Gaziosmanpasa Üniversitesi kampüsü içerisine kurulan ölçüm istasyonundan toplandı. Ölçüm istasyonu (N 40¹9'58.73") enlemi ve (E 36 $\left.29^{\prime} 0.28^{\prime \prime}\right)$ boylamına yerleştirilmiştir. Ölçüm direği 12 m yüksekliğinde olup üzerine 2 adet rüzgar hızı ölçüm sensörü ve 1 adet rüzgar yönü ölçüm sensörü yerleştirilmiştir.
Basınç, sıcaklık ve nem sensörleri direğin alt kısmına montaj edilen güç kutusuna yerleştirilmiştir. Sensörlerin ihtiyaç duyduğu enerji $10 \mathrm{~W}$ 'lık güneş panel ile sağlanmaktadır. Ayrıca diğer çalışmalarda kullanılmak üzere ölçüm direğinin tepesine AIR-X 400W rüzgar türbini yerleştirilmiştir.

Rüzgar hızı verileri 2017-2019 yılı boyunca 10'ar dakika aralıklar ile kaydedildi. Haftalık tahminlemelerde her ayın haftalarına karşılık gelen verilerin ortalaması alındı ve bir yıl için 48 veriye sahip bir çalışma seti oluşturuldu. Verilerin haftalık olarak değişimleri Şekil 1'de gösterilmektedir.

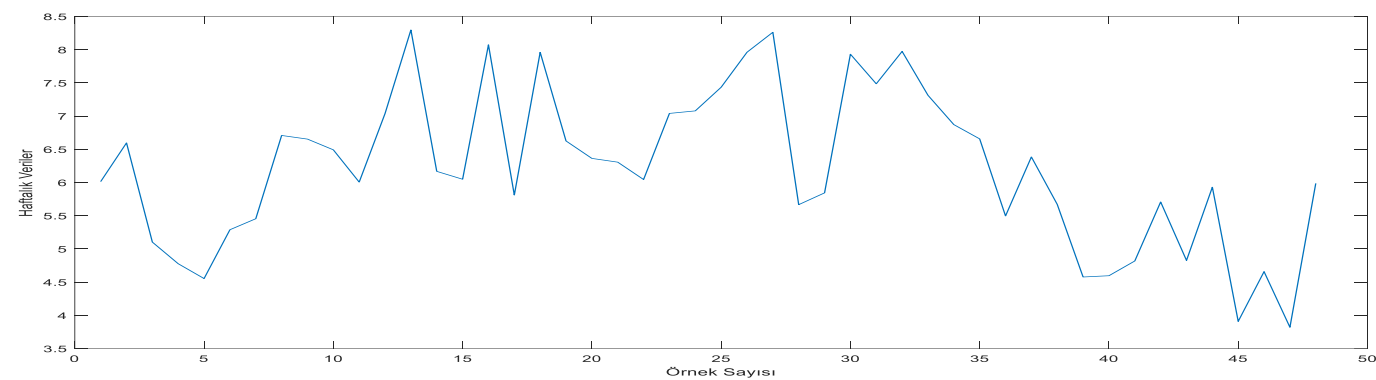

Şekil.1 Haftalık verilerin grafiği

Veri setindeki verilerin $75 \%$ eğitim verisi, $25 \%$ ise test verisi olarak kullanıldı. Verilere ait ortalama değer, maksimum değer, minimum değer ve standart sapmayı içeren istatistiksel bilgiler
Tablo 1'de listelenmektedir. Bu çalışmada önerilen EEMD-EWTPACF-BPNN modelinin etkinliğini doğrulamak için bir adımdan beş adıma kadar rüzgar hızı tahmin deneyleri yapılmıştır.

Tablo 1. Haftallk veri setlerinin istatistiksel bilgileri

\begin{tabular}{c|c|c|c|c}
\hline Veri seti & Ortalama & Maximum & Minimum & Standart Sapma \\
\hline Haftalık & 6.214 & 8.296 & 3.820 & 1.168 \\
\hline
\end{tabular}

\section{Sonuçlar}

Rüzgar hızı veri setlerinin özelliklerinin doğru ve güvenilir analizi, etkili bir tahmin modeli geliştirmek için kritik öneme sahiptir. Orijinal haftalık rüzgar hızı verileri, EEMD tekniği ile ayrıştırılmıştır. Şekil 2, azalan sırada orijinal rüzgar hızı serisinden çıkarılan farklı frekanslara sahip IMF'leri göstermektedir. IMF1-IMF4 ve R olmak üzere toplam 5 bileşen elde edilmiştir. Bu bileşenlerin en yüksek ve en düşük frekansları sırasıyla IMF1 ve R'dir. Şekil 3'de ana verilere en yakın alt veri kümesi olan IMF1, yeni bileşenlerine (EWT1, EWT2, EWT3) ayrıştırılmıştır.

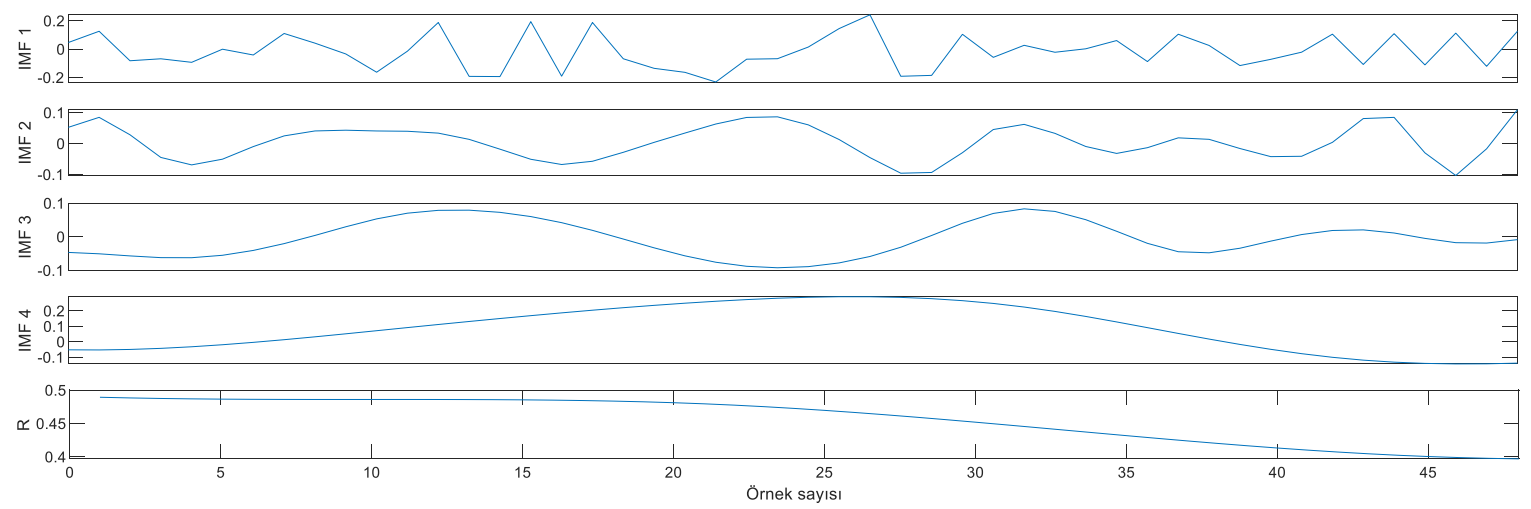

Şekil 2. EEMD tekniği ile elde edilen haftalık veriler için IMF'ler 

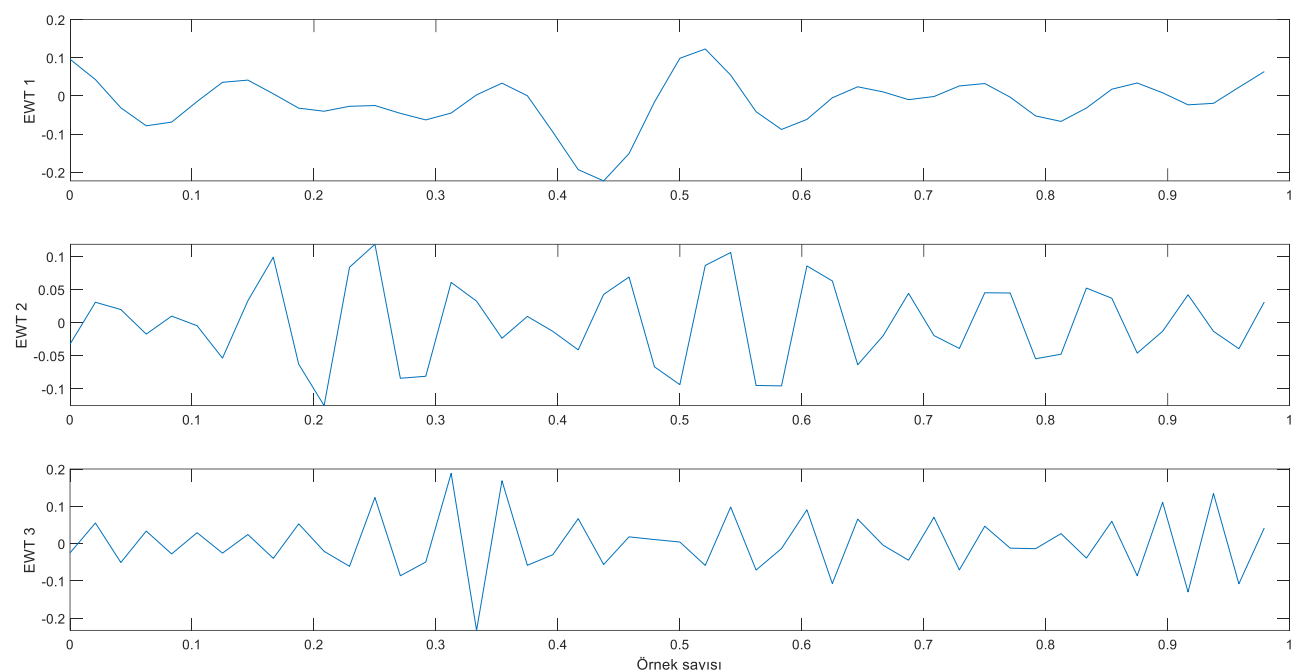

Şekil.3. Haftalık veri setinin IMF1 serisi, EWT aracıllğıyla ayrıștırıldı

Elde edilen IMF ve EWT alt serileri normalize işlemine tabi tutulur ve bu normalize edilmiş IMF'lerin kısmi otokorelasyon grafiği Şekil 4 ve 5'de gösterilmiştir. Grafikte PACF1 - PACF5, sirasiyla normalize IMF'nin PACF'unu, PACF6 - PACF8 ise EWT'lerin PACF'unu temsil etmektedir. Bölüm 2'de gösterilen girdi seçme yöntemine göre, Şekil 4 ve 5'deki çıkış değişkenleri $X_{t}$ ile gözlemlendiğinde, BPNN modellenmesi için girdi değişkenlerinin aşağıdaki gibi olduğu görülmektedir.

IMF1: $\left(X_{t-1}\right)$;
IMF2: $\left(X_{t-5}, X_{t-3}, X_{t-2}, X_{t-1}\right)$;
IMF3: $\left(X_{t-11}, X_{t-6}, X_{t-4}, X_{t-3}, X_{t-2}, X_{t-1}\right)$;
IMF4: $\left(X_{t-9}, X_{t-8}, X_{t-7}, X_{t-5}, X_{t-4}, X_{t-3}, X_{t-2}, X_{t-1}\right)$;

IMF5: $\left(X_{t-6}, X_{t-4}, X_{t-3}, X_{t-2}, X_{t-1}\right)$;

EWT1:

$\left(X_{t-12}, X_{t-11}, X_{t-10}, X_{t-9}, X_{t-8}, X_{t-7}, X_{t-6}, X_{t-5}, X_{t-4}, X_{t-3}\right.$, $\left.X_{t-2}, X_{t-1}\right)$

EWT2: $\left(X_{t-12}, X_{t-10}, X_{t-8}, X_{t-6}, X_{t-4}, X_{t-2}\right)$;

\section{EWT3:}

$\left(X_{t-12}, X_{t-11}, X_{t-10}, X_{t-9}, X_{t-8}, X_{t-7}, X_{t-6}, X_{t-5}, X_{t-4}, X_{t-3}\right.$, $\left.X_{t-2}, X_{t-1}\right)$;
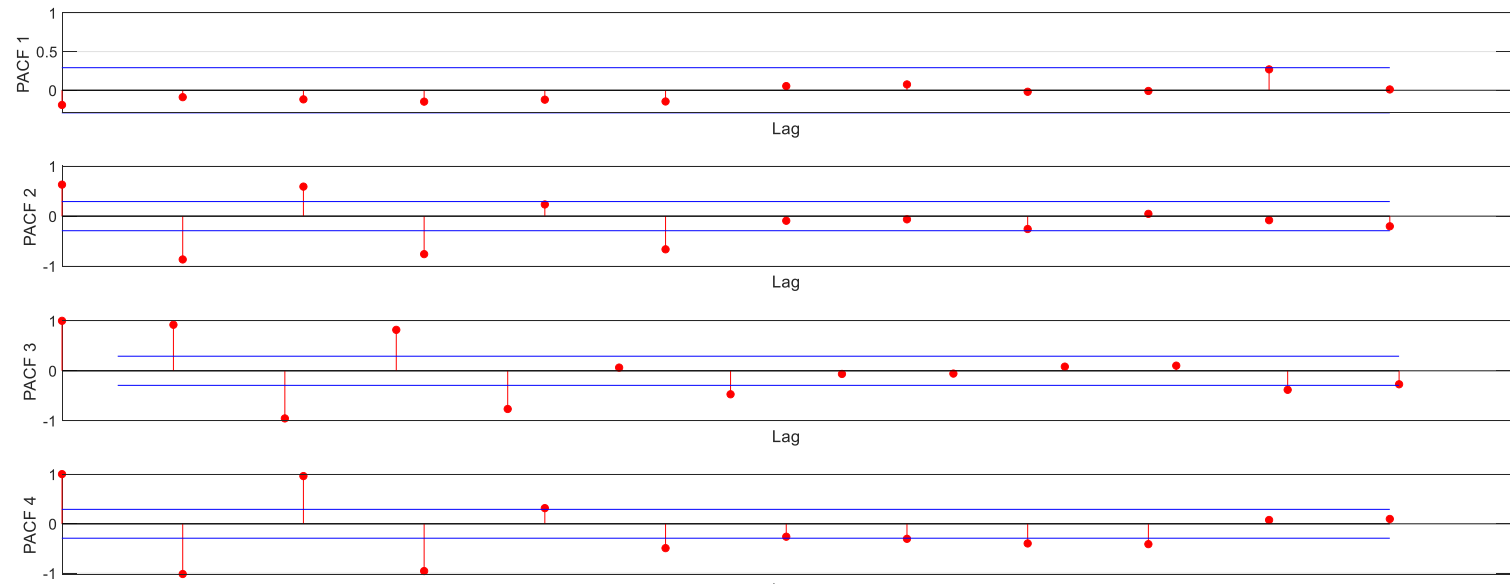

Lag

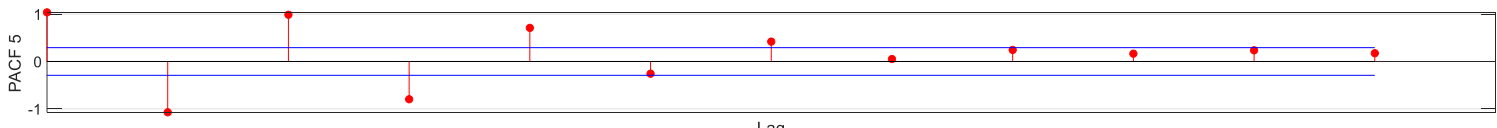

Lag

Şekil 4. EEMD üzerinden elde edilen PACF grafikleri 

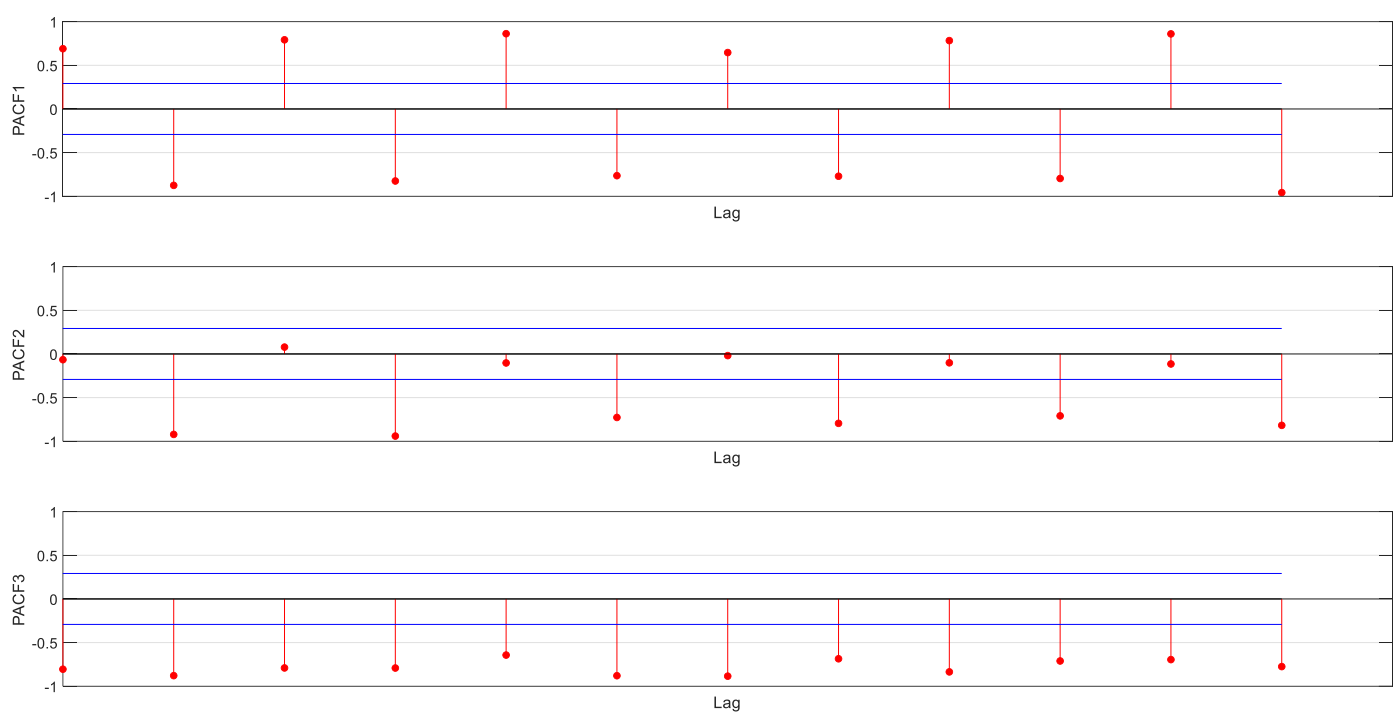

Şekil 5. EWT üzerinden elde edilen PACF grafikleri

Burada $X_{i}$ serisi, sırasıyla sekiz normalleștirilmiş seriyi temsil eder. $\mathrm{Bu}$ sekiz normalleştirilmiş seri BPNN yöntemi ile tahminlenmiştir. Tablo 2, tüm alt grupların bağımsız olarak
BPNN'a uygulanmasiyla elde edilen analizler için performans değerlendirmesini (RMSE, MAPE ve R²) göstermektedir.

Tablo 2. Ortalama haftalı rüzgar hızı tahminlemesinde tüm grupların performans karşılaş̧tırması

\begin{tabular}{|c|c|c|c|c|c|}
\hline Tahmin Adımları & Metrikler & EWT-BP & EEMD-BP & EEMD-EWT-BP & $\begin{array}{c}\text { EEMD-EWT-PACF-BP } \\
\text { (Hibrit Model) }\end{array}$ \\
\hline \multirow{3}{*}{ Adım 1} & $M A P E$ & 0.4578 & 0.9156 & 0.2787 & 0.2522 \\
\hline & $R^{2}$ & 0.5723 & 0.4197 & 0.2835 & 0.6928 \\
\hline & RMSE & 0.3427 & 1.1118 & 0.3432 & 0.3096 \\
\hline \multirow{3}{*}{ Adım 2} & MAPE & 0.6148 & 0.8259 & 0.3860 & 0.2094 \\
\hline & $R^{2}$ & 0.0381 & 0.1798 & 0.1158 & 0.7179 \\
\hline & RMSE & 0.4113 & 0.9524 & 0.4395 & 0.2848 \\
\hline \multirow{3}{*}{ Adım 3} & MAPE & 0.5381 & 1.0420 & 0.3407 & 0.1751 \\
\hline & $R^{2}$ & 0.0714 & 0.2014 & 0.5472 & 0.8612 \\
\hline & RMSE & 0.3593 & 1.2575 & 0.4188 & 0.2519 \\
\hline \multirow{3}{*}{ Adım 4} & MAPE & 0.5597 & 0.8421 & 0.2442 & 0.2389 \\
\hline & $R^{2}$ & 0.0374 & 0.2014 & 0.3050 & 0.3619 \\
\hline & RMSE & 0.3765 & 0.9132 & 0.3329 & 0.3142 \\
\hline \multirow{3}{*}{ Adım 5} & MAPE & 0.5497 & 0.7825 & 0.2766 & 0.2440 \\
\hline & $R^{2}$ & 0.4236 & 0.6460 & 0.7857 & 0.9173 \\
\hline & RMSE & 0.3812 & 0.8981 & 0.3594 & 0.3194 \\
\hline
\end{tabular}

Tablo 2 incelendiğinde, önerilen hibrit modelin birinci aşamadan beşinci aşamaya kadar tüm tahminler için tüm ölçümlerde en iyi sonucu verdiği görülmektedir. Önerilen modelin hata oranlarının tüm aşamalarda diğer alt gruplara göre daha düşük olduğu görülmektedir. Modellerin tüm adımlardaki elde edilen RMSE değerlerinin değişimi Şekil 6'da gösterilmektedir. Bununla birlikte tüm modellerde 5. adımda gerçekleştirilen tahminleme sonuçlarına göre rüzgar hızı değişimleri Şekil 7'de gösterilmektedir. 


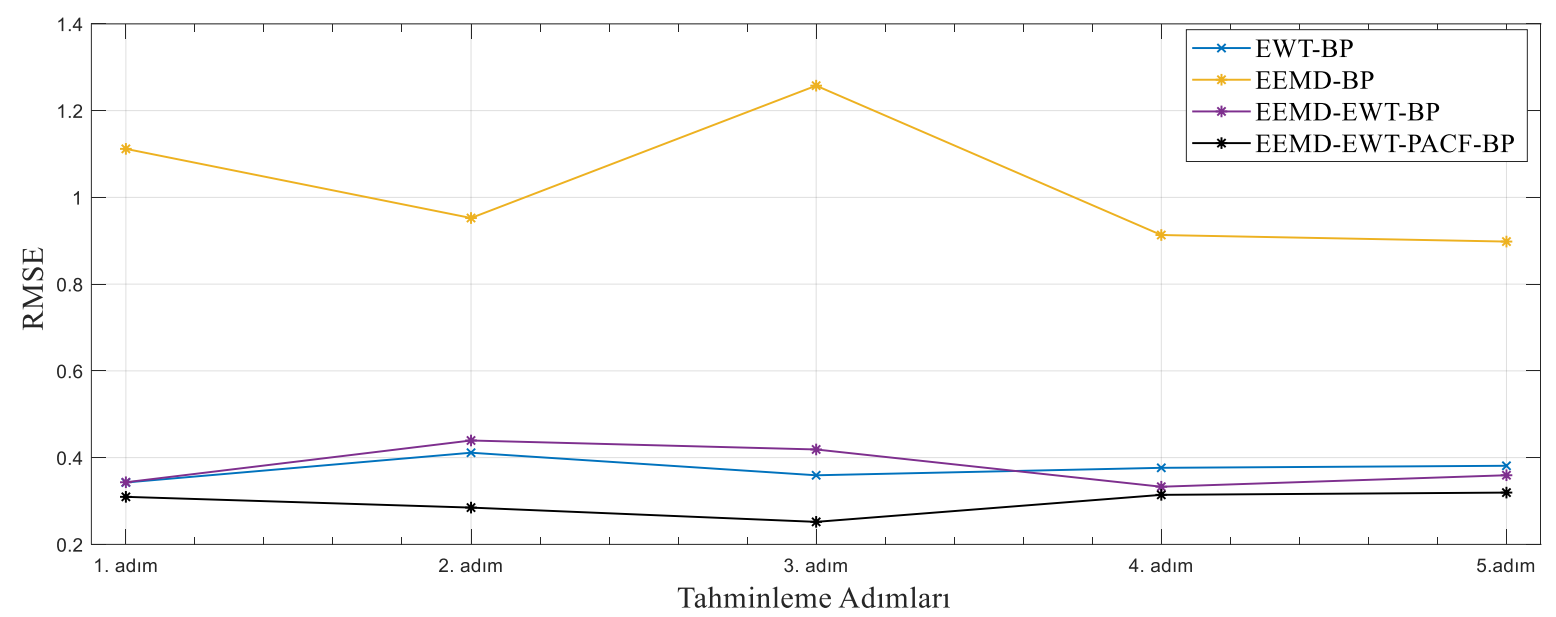

Şekil 6. Modeller arası RMSE değerlerinin değiş̧imi

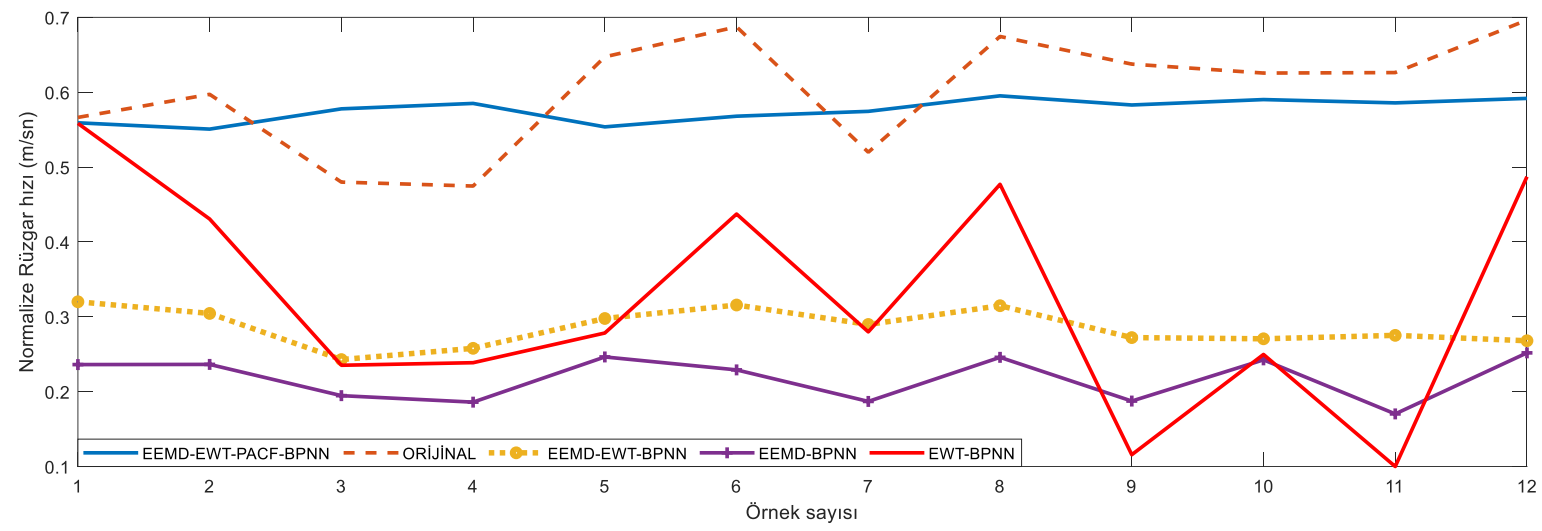

Şekil 7. 5. adımdaki tahmin sonuçlarına göre rüzgar hızlarının değisşimi

\section{Bulgular ve Tartışma}

Rüzgar enerjisinden maksimum verim elde etmek ve doğru planlama yapmak için rüzgar hızı tahmini çok önemlidir, bu nedenle yüksek doğruluk oranlarına sahip rüzgar hızı tahmin modelleri geliştirilmelidir. Orijinal rüzgar hızı zaman serileri tutarsız ve rastgele davranış sergiler ve bu nedenle birincil rüzgar hızı serilerinin kullanılması önemli hatalara yol açabilir. Hibrit ayrıştırma tabanlı yeni EEMD-EWT-PACF-BPNN modeli, çok adımlı rüzgar hızı tahmini için önerilmektedir. Ayrıştırma ve tahmin modeli, yeni veriler eklendiğinde önerilen modelde dinamik olarak ayarlanabilir ve güncellenebilir. Kurulan ölçüm istasyonunda rüzgar serileri ampirik olarak toplanmış ve bu sayede önerilen modelin uygulanabilirliği test edilmiş ve analizlerden elde edilen sonuçlar aşağıda sunulmuştur:

1- Önerilen EEMD-EWT-PACF-BPNN modeli, tüm alt gruplara (EEMD-BPNN, EWT-BPNN, EEMD-EWT-BPNN) kıyasla en iyi performansa sahiptir. Ayrıca önerilen modelin tek adımdan çok adıma kadar tüm tahmin prosedürleri için oldukça uygun olduğu görülmektedir.

2- Hibrit ayrıştırma yönteminin, rüzgar hızı tahmin kabiliyetinin iyileştirilmesi ve artırılması açısından tek ayrıştırma yöntemlerine (EEMD-BP, EWT-BP) göre daha iyi performans gösterdiği açıkça görülmektedir.

3- Sinir ağı girdileri, herbir alt fonksiyon önce normalizasyon işlemine sonrada PACF işlemine tabi tutularak belirlenmiştir.

Kısa vadeli rüzgar enerjisi tahminlerinin doğruluğunu artırmak için gelecekte davranış özelliklerine dayalı yeni birleşik tahmin yöntemleri önermek faydalı olacaktır. Bu çalışma ile ortaya konan modelin yol gösterici olması beklenmektedir. Önerilen hibrit model, yüksek hassasiyetli rüzgar hızı tahminleri için güvenilir, güçlü ve etkili olduğu kadar önemlidir. Veri madenciliği uygulamalarında da kolaylıkla kullanılabilir.

\section{Kaynakça}

Aghajani, A., Kazemzadeh, R., \& Ebrahimi, A. (2016). A novel hybrid approach for predicting wind farm power production based on wavelet transform, hybrid neural networks and imperialist competitive algorithm. Energy Conversion and Management, 121, 232-240.

Cadenas, E., \& Rivera, W. (2009). Short term wind speed forecasting in La Venta, Oaxaca, México, using artificial neural networks. Renewable Energy, 34(1), 274-278.

Emeksiz, C., \& Demir, G. (2018). An investigation of the effect of meteorological parameters on wind speed estimation using bagging algorithm. International Journal of Intelligent Systems and Applications in Engineering, 6(4), 311-321.

Gilles, J. (2013). Empirical wavelet transform. IEEE transactions on signal processing, 61(16), 3999-4010.

Guo, Z., Zhao, W., Lu, H., \& Wang, J. (2012). Multi-step forecasting for wind speed using a modified EMD-based artificial neural network model. Renewable Energy, 37(1), 241-249.

He, Q., Wang, J., \& Lu, H. (2018). A hybrid system for short-term wind speed forecasting. Applied Energy, 226, 756-771.

Hu, J., Wang, J., \& Ma, K. (2015). A hybrid technique for shortterm wind speed prediction. Energy, 81, 563-574. 
Huang, N. E., Shen, Z., Long, S. R., Wu, M. C., Shih, H. H., Zheng, Q., Yen, N. C., Tung, C. C., \& Liu, H. H. (1998). The empirical mode decomposition and the Hilbert spectrum for nonlinear and non-stationary time series analysis. Proceedings of the Royal Society of London. Series A: mathematical, physical and engineering sciences, 454(1971), 903-995.

Jiang, P., \& Li, C. (2018). Research and application of an innovative combined model based on a modified optimization algorithm for wind speed forecasting. Measurement, 124, 395-412.

Liu, H., Mi, X., \& Li, Y. (2018). Comparison of two new intelligent wind speed forecasting approaches based on wavelet packet decomposition, complete ensemble empirical mode decomposition with adaptive noise and artificial neural networks. Energy Conversion and Management, 155, 188-200.

Liu, H., Tian, H. Q, Liang, X. F., \& Li, Y. F. (2015). Wind speed forecasting approach using secondary decomposition algorithm and Elman neural networks. Applied Energy, 157, 183-194.

Liu, H., Wu, H., \& Li, Y. (2018). Smart wind speed forecasting using EWT decomposition, GWO evolutionary optimization, RELM learning and IEWT reconstruction. Energy Conversion and Management, 161, 266-283.

Ozbay, Y., \& Karlik, B. (2002). A fast training back-propagation algorithm on windows. Proceedings of the Third International Symposium on Mathematical \& Computational Applications,

Peng, T., Zhou, J., Zhang, C., \& Zheng, Y. (2017). Multi-step ahead wind speed forecasting using a hybrid model based on two-stage decomposition technique and AdaBoost-extreme learning machine. Energy Conversion and Management, 153, 589-602.

Qian, Z., Pei, Y., Zareipour, H., \& Chen, N. (2019). A review and discussion of decomposition-based hybrid models for wind energy forecasting applications. Applied Energy, 235, 939-953.

Ren, C., An, N., Wang, J., Li, L., Hu, B., \& Shang, D. (2014). Optimal parameters selection for BP neural network based on particle swarm optimization: A case study of wind speed forecasting. Knowledge-Based Systems, 56, 226-239.

Santhosh, M., Venkaiah, C., \& Kumar, D. V. (2018). Ensemble empirical mode decomposition based adaptive wavelet neural network method for wind speed prediction. Energy Conversion and Management, 168, 482-493.

Sun, N., Zhou, J., Chen, L., Jia, B., Tayyab, M., \& Peng, T. (2018). An adaptive dynamic short-term wind speed forecasting model using secondary decomposition and an improved regularized extreme learning machine. Energy, 165, 939-957.

Şenkal, S. (2014). Rüzgar Hızı Tahmin Yöntemleri - Örnek Bir Uygulama Ondokuz Mayıs Üniversitesi. Ondokuz Mayıs Üniversitesi, Fen Bilimleri Enstitüsü, Yüksek Lisans Tezi.

Tan, M. (2020). İkincil ayrıştırma tekniği kullanarak yapay sinir ağ1 temelli çok adımlı rüzgar hızı tahmini. Tokat Gaziosmanpaşa Üniversitesi, Fen bilimleri Enstitüsü, Yüksek Lisans Tezi.

Tascikaraoglu, A., \& Uzunoglu, M. (2014). A review of combined approaches for prediction of short-term wind speed and power. Renewable and Sustainable Energy Reviews, 34, 243-254.

Torres, M. E., Colominas, M. A., Schlotthauer, G., \& Flandrin, P. (2011). A complete ensemble empirical mode decomposition with adaptive noise. 2011 IEEE international conference on acoustics, speech and signal processing (ICASSP).

Wang, H., \& Zhao, W. (2009). Arima model estimated by particle swarm optimization algorithm for consumer price index forecasting. International Conference on Artificial Intelligence and Computational Intelligence.

Wang, S., Zhang, N., Wu, L., \& Wang, Y. (2016). Wind speed forecasting based on the hybrid ensemble empirical mode decomposition and GA-BP neural network method. Renewable Energy, 94, 629-636.

Wu, Z., \& Huang, N. E. (2009). Ensemble empirical mode decomposition: a noise-assisted data analysis method. Advances in adaptive data analysis, 1(01), 1-41.

Xiao, L., Wang, J., Dong, Y., \& Wu, J. (2015). Combined forecasting models for wind energy forecasting: A case study in China. Renewable and Sustainable Energy Reviews, 44, 271-288.

Zhang, W., Qu, Z., Zhang, K., Mao, W., Ma, Y., \& Fan, X. (2017). A combined model based on CEEMDAN and modified flower pollination algorithm for wind speed forecasting. Energy Conversion and Management, 136, 439-451. 